gesetzen, in die das politische Gewicht ihrer Klassenfeinde - der Arbeiterparteien - mit eingegangen war.

Die objektiven $Z$ wänge bestehen fort. Der pure Wille zu einer neuen Systematik droht zu Bestrebungen zu gerinnen, die - wie weiland der Senatspräsident a. D. Baumbach - Gesetze vor der Verabschiedung einem Gesetzgebungsamt vorlegen möchten. Dort kann man sie unter dem Vorwand der formalen Aufmöbelung auf bornierte juristische Sozialideale hin umformen. Ernst Wolfs Blindheit gegenüber den Bestimmungsgründen der Entwidslung des Lehrstoffs hat ihre Entsprechung dessen Konsumenten gegenüber.

Die Studenten

Ihnen mangelt es immer mehr an "Kenntnissen * und "Denkschulung *. Das liegt an der Schule, die immer mehr Abiturienten mit zunehmend geringeren Leistungen ausstößr. Daran ist das die Hürden herabsetzende "Kultusministerium" schuld, denn $*$ Latein $\approx$ mit seinen hohen Anforderungen ans systematische Denken wird,.. zugunsten diffuser Fächer wie zum Beispiel, Gemeinschaftskundee reduziert..... Lateinlernen als eine der Grundvoraussetzungen einer neuen Systematik der Rechtswissenschaft? - das kann nur ein Witz sein. Zugunsten der umstrittenen Errungenschaft ,Gemeinschaftskunder ist zu sagen, daß - wenn dieses $>$ Fach، seinem Anspruch gemäß betrieben würde - es den Vorteil böte, die Wolfsche Blindheit heilbar zu machen. Doch Wolf insistierr: Ihm erzeugt die Schule zu viele undisziplinierte und leistungsschwache Abiturienten; es wird dort sogar »diskutierr «, natürlich sohne hinreichende Grundlagen «. Kein Wunder, daß man sich dann an der Universität schwer tut! *Bei solchen Verhältnissen kann es nicht überraschen, daß unter den Studenten eine Mißstimmung verbreitet ist, die von der geschickt arbeitenden Propaganda linksradikaler Gruppen für deren destruktive politische und igesellschaftspolitisches, das heißt marxistisch-gesellschaftsrevolutionäre Ziele erfolgreich ausgenutzt wird «.

Ernst Wolfs Analyse erklärt nichts. Ihre Geistesart ist symptomatisch für eine bestimmte Gattung von Professoren, deren Herrschaftsinteresse sie recht dilettantisch zu verschleiern sucht.

Anna Jobow

\title{
Ein Flugblatt im besonderen Gewaltverhältnis
}

Dem Assessor im Lehramt Heinz Lüdde, tätig am Kreisgymnasium in Heusenstamm (Landkreis Offenbach), wurde am Ir. November 1968 vom Regierungspräsidenten in Darmstade aus zwingenden dienstlichen Gründen die Führung seiner Dienstgeschäfte verboten ( $\$ 74$ Hessisches Beamtengesetz). Die sofortige Voliziehung der Suspendierung lag im öffentlichen Interesse ( $\$ 80 \mathrm{Abs.} 2 \mathrm{Ziff} .4$ Verwaltungsgerichtsordnung). Dieses wird repräsentiert durch zwei katholische Kirchenvorstände in Heusenstamm und einige Elternbriefe. Die zwingenden dienstlichen Gründe sind die Verteilung eines Flugblattes an Schüler einer 9. Klasse des Gymnasiums, die Erklärung des Assessors, die Lehrpläne für Schulen des Landes Hessen seien für ihn nicht verbindlich (bestritten) und die Veranlassung von Schülern, Resolutionen zu verfassen, in denen die Verhaltensweise bzw. der Unterricht älterer Kollegen kritisiert wurde (bestritten). $D_{a}$ die 
beiden letztgenannten Gründe schon eine gewisse Zeit zurüdklagen, ohne daß der Regierungspräsident einschritt, drängt sich der Schluß auf, Assessor Lüdde sei wegen der Verteilung eines Flugblatts im Unterricht suspendiert worden. Lüdde wurde vernommen. Ihm wurde eröffnet, es sei beabsichtigt, das Beamtenverhältnis auf Widerruf zu widerrufen ( $\$ 42 / 43 \mathrm{HBG}$ ).

Das Flugblatt, herausgegeben vom Sozialistischen Lehrerbund und einer sozialistischen Schülerorganisation in Frankfurt (AUSS) und in Frankfurt, Darmstadt, Wiesbaden und anderen hessischen Städten z. T. von Lehrern in der Schule verteilt, fordert alle Schüler und Schülerinnen ab I4 Jahren auf, den Religionsunterricht massenhaft $z u$ verlassen. Begründung: Religionsunterricht macht unkritisch, nörigt zur Anpassung und ist tendenziös (Informationsaussparungen: kirchlicher Antisemitismus, triebfeindliche Sexualmoral, Diffamierung der Frau, Inquisition, Mission und Kolonialismus, religiöse Rechtfertigung der Klassenstruktur der Geselischaft); religions-ideologiekritische Arbeiten (Feuerbach, Marx, Nietzsche, Freud, Bloch) werden vorenthalten. Literaturhinweise sind auf dem Flugblart angegeben (Freud, Kahl).

Die Suspendierung eines Lehrers mit dem Ziel der Entlassung aufgrund der Verteilung des Flugblatts stellt sich funktional als der Versuch der Unterdrükkung von freier Meinungsäußerung und Informationsfreiheit dar. Die Maßnahme ist ein Ansatzpunkt gesellschaftlicher Formierung, die durch die Aufrechterhaltung von Tabus herrschende gesellschaftliche Zustände selbst sanktionieren und stabilisieren soll. Mitrel dieser Formierung sind scheinbar neutrale, rechtliche Instrumentarien. Inhaltlich politische Akrionen werden in formalrechtliche Kategorien gefaßr, die sich dann den Anschein objekriver Rechtswahrung (Friedenswahrung) geben, in Wirklichkeit aber nichts anderes sind als die Umsetzung politischer Ziele. Dabei funktioniert dieser Umsetzungsprozeß so, daß von vornherein juristisch die inhaltliche Diskussion des Flugblatts (die gerade beabsichtigt war) ausgeschlossen wird. Die Suspendierung wird gestützt auf die Verletzung beamtenrechtlicher Treuepflichten, die dahin konkrecisiert werden, daß ein Lehrer solche Flugblätter nicht während des Unterrichts ohne Genehmigung verteilen darf (anders kann der Vorwurf nicht verstanden werden). Beamtenrechtliche Treuepflichten des Lehrers dem Staat gegenüber werden so als Mittel politischen Herrschaftskampfes gewender. Diese Treuepflichten sind Inhalt des durch die Verleihung der Beamteneigenschaft an den Lehrer begründeten besonderen Gewaltverhältnisses. Historische Funktion des besonderen Gewaltverhältnisses war es, durch die Schaffung eines »rechtsfreien* Raumes (bezogen auf den parlamentarischen Gesetzgeber) dem Monarchen auch im Konstitutionalismus weite Reservate absolutistischer Machtfülle zu bewahren (H. H. Rupp, Grundfragen der heutigen Verwaltungsrechtslehre S. 77 ff mit weiteren Literaturangaben). Wenn heute die juristische Diskussion um dieses Institut auf eine Rechtsschutzproblematik redressiert wird, so zeigt dies, wie wenig demokratische Verfassungsordnung den "Innenbereich« der gesamten Verwaltung verändert hat. Die Eingrenzung verbürgter Grundfreiheiten bleibt garantiert, die Auslösung eines Demokratisierungsprozesses wird verhindert. Durch Maßnahmen, wie die hier referierte, werden in der Schule, die nach ihrem Selbstverständnis Ort der Erziehung zu einem demokratischen Bewußtsein sein soll, Informationen und Diskussion über wissenschaftliche Erkenntnisse unterdrüdkt oder doch wesentlich erschwert. Tolerantes Verhalten ais Ergebnis eines Lernprozesses kann auf diese Weise nicht vermittelt werden.

Das besondere Gewaltverhältnis zeigt seine repressive Wirkung deutlich beim Beamten auf Widerruf, also meist jungen Lehrern, die am ehesten noch neue 
Unterrichtsinhalte und -methoden in die Schule einzubringen vermögen. Der Widerruf ihres Beamtenverhältnisses kann aus »irgendwelchen sachlichen Gründen * erfolgen (H. J. Wolff, Verwaltungsrecht II, I962, S. 36 I; vgl. z. B. Ordnung der Laufbahn im Lehramt an höheren Schulen vom April I954 - Amtsblatt [Hessen] S. I 44). Die Abhängigkeit der jungen Assessoren und damit der $Z$ wang, sich althergebrachten Unterrichtsmodellen zu fügen, wird so offenkundig. Wenn es dann in der juristischen Literatur heißr, der Lehrer sei in der Methode seines Unterrichts frei (I. v. Münch, Die pädagogische Freiheit des Lehrers, Deutsches Verwaltungsblatt 1964,789 ff, 795), so kann solche Meinung nur unter Abstrahierung sozialer und gesellschaftlicher Abhängigkeitsverhältnisse aufgestellt werden.

Der ${ }_{\text {Fall }}$ des Assessors Lüdde mag als Einzelfall angesehen werden. Seine Bedeutung gewinnt er vor dem Hintergrund einer Gesellschaft, die oppositionelles politisches Engagement mit rechtlichen Mitteln auszuschalten versucht.

Dieter Hart

\section{Justiz und NS-Verbrechen}

Vor kurzem ist der erste Band der insgesame 2r Bände der Serie "Justiz und NS-Verbrechen " erschienen, einer Sammlung der in der Zeit vom 8. 5. 1945 bis zum I. I. 1966 ergangenen rechtskräftigen Urteile der deutschen Land- und Rechtsmittelgerichte in Strafsachen wegen nationalsozialistischer Tötungsverbrechen 1 .

Es erscheint mir nützlich zu sein, den Zweck der Sammlung und die Weise ihres Zustandekommens zu erläutern, insbesondere den wohl etwas merkwürdig anmutenden Umstand zu erklären, daß gerade diese Sammlung, sei es auch unter starker deutscher Beteiligung ${ }^{2}$, an der Universität von Amsterdam vorbereitet wird und bei einem niederländischen Verlag erscheinen muß.

Um den letzten Punkt gleich vorwegzunehmen: Zwar wurde die Bearbeitung von der Alexander-von-Humboldt-Stiftung und wird sie jetzt von der Deutschen Forschungsgemeinschaft und von der niederländischen Forschungsgemeinschaft $* Z$. W. O. subventioniert, ein Druckeostenzuschuß stand und steht jedoch nicht zur Verfügung. Von allen - zuerst in der Bundesrepublik, später auch in den Niederlanden - angegangenen Verlagen war nur einer bereir, das Risiko selbst voll zu übernehmen: die Verlegergemeinschaft "Associated Publishers

1 Gemeint sind damit Mord und Totshlag, ferner Körperverletzung, Freiheitsberaubung, Rechrs beugung, Kriegsverbrechen und Verbrechen gegen die Menschlichkeit, jeweils $\gg$ mit Todesfolgex. $\mathrm{Dabei}$ ist nichr darauf abgestellt, daß es zu einer Verurteilung gekommen ist. Maßgebend für eine Veröffentichung in der Sammlung ist vielmehr der Gegenstand der Anklage. Ferner komme es nicht darauf an, ob die Todesfolge in einem nach dem Tatbestand des betreffenden Strafgesetzes relevanten Kausaizusammenhang mit der strafbaren Handlung steht. Bei einigen Verbrechen könnte ein solcher Zusammenhang auch gar niche festgestellt werden, da das Geriche sich darüber nicht ausläßr, weil der Todeserfolg, z. B. bei den Verbrechen gegen die Menschlichkeir, nicht $z$ den gesetzlichen Tatbestandsmerkmalen gehört.

2 Der Redaktion gehören deutscherseits Prof. Dr. Karl Dietrich Bracher/Bonn und Prof. Dr. Hans Heinrich Jeschede/Freiburg an. Auch Dr. Fritz Bauer hat als Redaktionsmitglied bis zu seinem Tode an den Vorbereitungen starken Anteil genommen. Die Bearbeitung erfolgt maßgeb. lich durch die deutsche Juristin Frau A. L. Rüter-Ehlermana. 